Check for updates

1 The BMJ

Cite this as: BMJ 2021;375:n2978 http://dx.doi.org/10.1136/bmj.n2978 Published: 02 December 2021

\section{The eyes have it: getting the gist of covid-19 transmission}

\author{
A new interactive graphic, published by The $B M$ J, is designed to help people to decide how best to \\ protect themselves and others from covid-19. Will Stahl-Timmins explains why data visualisation \\ can help us understand complex problems, and what inspired him and his co-authors to create this \\ graphic
}

\section{Will Stahl-Timmins data graphics designer}

Many words have been written and numbers collected about covid-19 over the past two years, but visualisation can further enhance our understanding of how the virus can be transferred between us. We've been told we need to wash our hands, wear masks, open the window, stay further apart, and not hug granny unless she's been fully vaccinated. But understanding the relative importance of these measures in different situations and how they combine is challenging. What if I'm singing? Eating? Exercising? Outside? We thought that creating an interactive visualisation could help us show our audience the relative importance of different measures, although it was a challenge to present uncertain information in a way that would be easy to understand. ${ }^{1}$

One of the main benefits of data visualisation is that it makes use of the human visual processing system to absorb large amounts of information quickly. ${ }^{2}$ The reason that line and bar charts are so widely used in science is that they allow us to get the gist of a data series in just a few seconds, which would be impossible when looking at the raw numbers. We wanted to use this visual processing capability to show people the relative importance of different disease transmission routes, thereby revealing the "invisible" to the naked eye.

Data visualisation expert Alberto Cairo says that "We should certainly do good with data, but only after we've thoroughly made sure that our data is good." 3 The dataset on which we based our visualisation was painstakingly collected by members of a sub-group of the UK government's Scientific Advisory Group for Emergencies: the Environment and Modelling Group (SAGE-EMG). They elicited opinions from 26 experts to collect the thousands of data points needed for all scenarios and mitigation measures in the visualisation. While their methods were robust, we still know relatively little about covid-19, so the resulting model has many dimensions of uncertainty-such as disagreement between experts, quality of underlying evidence, and the range of variable situational elements like moisture levels in the body and atmosphere.

Presenting uncertain data are always challenging. There was much discussion at the recent Information+conference about the concept of "seamfulness" (the opposite of seamlessness) in visualisation, inspired by a talk from Nicole Hengesbach, a PhD student at the University of Warwick. One problem with visualising uncertain data are that polished visuals and beautiful design can lull the viewer into a false sense of security. The concept of seamfulness in design aims to expose the rough edges in data, highlighting uncertainty and gaps in knowledge. To this end, we made the unusual choice of a colour scale to present the likelihood of disease transmission. Colour is normally used to present lower-order data like categories in most data visualisations, as other elements such as position and size of objects are easier to grasp. In this case, we intentionally made it harder for the viewer to focus on exact values, instead encouraging them to get a "sense" of the relative differences.

We also showed several aspects of the uncertainty of the data in a more specific way. There are blue information buttons all around the graphic that can be clicked on to bring up a window we called the "geek interface" in development. This presents more interested and engaged users with a confidence interval for the values elicited from experts at each point in the visualisation, the experts' assessment of the quality of the underlying evidence, and a text description of the main uncertainties for this part of the graphic.

The visualisation allows a viewer to explore multiple different options by changing scenarios and different protective measures. By presenting the information on risk of transmission visually, encoded into a colour scale, each change made by the user can instantly be seen throughout the network of pathways. This allows people to understand not just what can be done, but which are the most effective things to do in a given situation, according to the experts consulted. This ability to experiment with different scenarios and protective measures will be particularly important as the conditions around covid-19 continue to change and develop, with vaccination rollouts, and new variants like delta and omicron. Hopefully this visualisation will help to clear the clouded air of confusion around how best to prevent the transmission of covid-19, allowing us to see more clearly the most important measures to use in different situations.

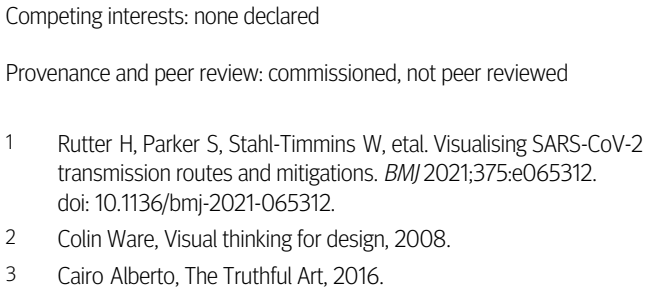

